\title{
Meta-Analysis of the Variables Related to Subjective Well-being of Youth
}

\author{
Sung-Joo Kang ${ }^{1}$ and Hyoun-Yong Kwon ${ }^{2}$ \\ Hyupsung Univ, Korea \\ 2khyong@hanmai.net
}

\begin{abstract}
This study aims to provide basic data for youth's healthy psychosocial growth through the meta-analysis of protective factors and risk factors related to "the subjective well-being" of youth (middle \& high school students). A total of 66 articles, including 44 theses and 22 journal papers were finally selected for the meta-analysis. Here are the results of the study. First, the effect size of protective factors of youth's "the subjective well-being" was mid-level, similar to that of individual characteristics variables and family variables and the effect size of social variables was relatively lower than the mid-level. Second, the effect size of risk factors of youth's "the subjective well-being" was all below mid-level, and the effect size of family variables was highest. The effect size of individual characteristics variables was in order of mental-health, neurotic personality, and family variable's instability attachment were relatively high. Based on these results, it is meaningful that this study provided the practical data to develop education, and group counseling program for improving "the subjective well-being" and seek how to approach the improvement of "the subjective wellbeing" in personal counseling.
\end{abstract}

Keywords: The subjective well-being, Youth (middle \& high school students), Metaanalysis, Protective factors, Risk factors

\section{Introduction}

"The subjective well-being" is another expression of happiness, and high "The subjective well-being" is a condition where a person is more satisfied with his life and experiences more positive emotions than negative emotions [1]. Everyone wants to be happy, and every parent is eager for their children's happiness. Korean parents have been striving for the happiness of their children based on the values they consider to be right, and the government has also continued to implement education and welfare policies for the happiness of children and youth. However, if you ask Korean youths, "Are you happy?" I wonder how many of the youths will answer "Yes, happy."

"The subjective well-being" is an emotional reaction of positive thoughts and feelings that an individual feels in everyday life [2]. So how do each individual have a cognitive, emotional, or attitude toward the situation in which he or she is facing? Or where do you put your weight on life now? This depends on the influence of subjective aspects such as [3]. In other words, "The subjective well-being" is an integrated judgment about life that reflects stable emotions about one's life based on the individual's subjective experience [4].

Article history:

Received (October 12, 2019), Review Result (November 19, 2019), Accepted (December 22, 2019) 
Therefore, when a question about "The subjective well-being" is given, the quality of life, or feelings of well-being or satisfaction, reflected in the responses of the adolescents exist within the experiences of each young person, and the individual's cognitive and emotional integration of their life is It is evaluation.

The 'subjective happiness index' of Korean children and youths in 2018 recorded a standard score of 94.6 [5]. According to the report, the 'Satisfaction with School Life' score increased significantly from 29.4 points in 2009 to 43.7 points in 2018 , as a direct reason for the increase in the subjective happiness index of adolescents. However, responses related to fit and adaptation at school are the lowest among OECD countries, indicating that school life satisfaction scores are far from relationship satisfaction. In the report, the subjective happiness scores of students who selected relational factors were higher than those of students who selected material factors. As the grades in Korea, the rate of responding to material values rather than relational values increases. The rate at which they felt happy decreased as their grade increased.

People with high "The subjective well-being" are very good at solving problems, have meaningful social relationships, and are very physically and mentally healthy, such as adapting to stress situations [6]. It also shows positive results in various areas of life, such as marriage, friendships, work life and job achievement, economic income, mental health and physical health [7]. This supports the claim that "The subjective well-being" has a stable character throughout life, and that it has a positive effect on many areas of human life.

Adolescence is an important time when there is a rapid physical change and maturation of thought, and it is not only sensitive to peer recognition, but also to form psychological independence and identity [8]. Adolescents' growth and development and "The subjective well-being" are influenced through a multi-step process in which their families and communities are combined [9]. Adolescents are in the stage of developmental transitions in which independence and dependence intersect, and their sense of well-being is in serious crisis because they are less coping and problem-solving than adults [10]. Their low "The subjective well-being" leads to maladjustment and further to life and social maladjustment. On the other hand, high "The subjective well-being" has a significant correlation with current and future social life adjustments [11].

The results of this study are intended to provide a basis for developing counseling, education, and group counseling programs to improve "The subjective well-being" for young people, as well as seeking and supporting youth counseling methods. It is also expected to be used as a basis for various adaptive interventions to improve "The subjective well-being" of adolescents at school sites. Furthermore, I would like to suggest the direction of follow-up research related to the subjective well-being of adolescents.

The purpose of this study is as follows. First, what is the effect size of "The subjective well-being" protection factors of adolescents? Second, what is the effect size of "The subjective well-being" risk factors for adolescents?

\section{Research method}

\subsection{Methods and procedures for selecting research targets}

The paper search method was searched by the key search terms in each institution's website and cross-checked by each institution. In order to search the data related to the subjective well-being of adolescents, 391 papers were searched with the main terms 'youth' and 'the subjective well-being'. After that, the study was limited to middle school and high 
school students for the second time, and thesis related to subjective well-being was selected for analysis. Finally, the thesis selected for analysis was 44 dissertations and 22 journals. A total of 66 papers were analyzed. The target material was used.

\subsection{Data analysis}

The collected paper on subjective well-being produced a separate coding manual to identify the characteristics. Coding of 66 papers to be analyzed was classified into sample size $(\mathrm{N})$, publication year, publication status, correlation coefficient ( $\mathrm{r}$ value), and categorical variables (middle and high school students, measurement tools). Coding was carried out through discussion between the researcher and two Ph.D. students, and the classification of sub-variables was conducted with the advisor. After the coding was completed in accordance with the manual, a total of three researchers reviewed the coding materials for review, one doctoral program in statistics and one advisory professor. The coded data were analyzed using CMA 2.0 program (Comprehensive Meta-Analysis Ver.2).

\section{Result of research}

\subsection{Protective factors associated with "The subjective well-being" of adolescents}

All of the personal characteristic variables $(\mathrm{ESr}=.351)$ were above the moderate level of effect, and there was a significant average effect in the 95\% confidence interval. Elasticity $(\mathrm{ESr}=.420)$, optimism $(\mathrm{ESr}=.457)$, desire for basic psychology $(\mathrm{ESr}=.416)$, and efficacy $(\mathrm{ESr}=.403)$ are large in terms of the interpretation of effect sizes presented by Cohen. A high effect size approaching the level was found, and the mean effect was found to be significant in the 95\% confidence interval. Self-esteem (ESr=.356), psychological well-being $(\mathrm{ESr}=.333)$, friendly personality factors $(\mathrm{ESr}=.312)$, functional self-control $(\mathrm{ESr}=.309)$, and positive emotions $(\mathrm{ESr}=.303)$ were Cohen $(\mathrm{ESr}=.303)$. According to the effect size analysis criteria suggested by 1988), it corresponds to an effect size of more than a moderate level and a significant average effect exists in the 95\% confidence interval. Cultural propensity $(\mathrm{ESr}=.215)$ and sports participation $(\mathrm{ESr}=.211)$, based on the interpretation of the effect size presented by Cohen, corresponded to sub-medium effect sizes and were significantly significant at the $95 \%$ confidence interval. Average effects were found to exist. Academic scores $(E S r=.159)$ and health status $(E S r=.157)$ are based on Cohen's interpretation of the magnitude of the effect, which corresponds to more than a small level of effect, and is significantly averaged at $95 \%$ confidence intervals.

Family variables $(\mathrm{ESr}=.348)$ were moderate to moderate, with significant mean effects at 95\% confidence intervals. Empathy $(\mathrm{ESr}=.432)$ and affective/acceptive parenting attitudes $(\mathrm{ESr}=.404)$ are based on Cohen 's interpretation of the effect size, which corresponds to a high effect size approaching a large level, 95\%. The mean effect was found to be significant in the confidence interval. Family health $(\mathrm{ESr}=.323)$ and stable attachment $(\mathrm{ESr}=.304)$, based on Cohen's interpretation of effect size, correspond to moderate to moderate effect sizes, and are significantly averaged at $95 \%$ confidence intervals. The effect appeared to exist. The economic level $(\mathrm{ESr}=.236)$ corresponds to the effect size analysis criteria presented by Cohen, which is below the medium level, and has a significant average effect in the $95 \%$ confidence interval.

All social variables $(\mathrm{ESr}=.295)$ were below the moderate level of effect, with a significant average effect in the $95 \%$ confidence interval. Both social support $(\mathrm{ESr}=.321)$ and positive interpersonal relationships (ESr=.264) are based on Cohen's interpretation of the magnitude 
of the effect, which is below the moderate level of magnitude and significantly higher in the 95\% confidence interval. Average effects were found to exist.

\subsection{Risk factors associated with "The subjective well-being" of adolescents}

The overall personality variables of the risk factors $(\mathrm{ESr}=-.238)$ were below the medium level of effect, and there was a significant mean effect in the $95 \%$ confidence interval. Mental health problems (ESr=-.448) are based on Cohen's interpretation of the magnitude of the effect, which is a high effect size approaching a large level, with significant mean effects at the $95 \%$ confidence interval appear. The neurotic personality factor $(\mathrm{ESr}=-.316)$ is based on Cohen's interpretation of the effect size, which indicates that the effect size is at least moderate, and that the mean effect is significant in the 95\% confidence interval. Stress (ESr=.295), Indecision ( $\mathrm{ESr}=-.237)$, Negative Emotions ( $\mathrm{ESr}=-.204)$, Internet Addiction ( $\mathrm{ESr}=-$ $.162)$, Perfectionism (ESr=-.162), Understated The excess self-control (ESr=-.124) was all based on Cohen's interpretation of the effect size, indicating that the effect size was below the medium level and there was a significant average effect in the $95 \%$ confidence interval.

Family variable of risk factors (ESr=-.208) was below the moderate level of effect, with a significant mean effect in the $95 \%$ confidence interval. Sibling relations were statistically insignificant because confidence intervals included zero. Both unstable attachment (ESr=.281) and coercive and controlled parental attitudes (ESr=-.64) are based on the interpretation of effect size presented by Cohen. The mean effect was found to be significant in the interval.

The overall social variables of risk factors $(\mathrm{ESr}=-.213)$ were below medium level of effect, and there was a significant average effect in $95 \%$ confidence interval. Negative interpersonal relationships $(\mathrm{ESr}=-.168)$ and social conflict $(\mathrm{ESr}=-.244)$ both correspond to the effect size interpretation criteria presented by Cohen, which is below the moderate level of magnitude, with $95 \%$ confidence intervals. Significant average effects were found.

\section{Conclusion \& Discussion}

First, the overall effect size of protection factors related to adolescent's "The subjective well-being" was above the middle level, and the effect size of personal characteristic variables was higher than that of family variables or social variables. In personal characteristics, optimism, resilience, efficacy, and basic psychological desire were found to have high effect size. Family variables were more effective in affective and receptive parenting attitudes on how well the family functions and the psychological and emotional communication is positive than the physical aspects of the home. Social variables were moderate.

Second, the overall effect size of risk factors negatively related to "The subjective wellbeing" of adolescents was below the middle level, and the effect size of family variables was higher than that of personal characteristics and social variables. For young people, the home is a source of happiness and a place where stable emotions and self-esteem are nurtured. Affection and support provided at home can be expected to protect against a variety of risk factors. Adolescents who have established a stable attachment feel satisfaction and joy from the belief that they and others are valued and that they deserve to be loved. Affectionate and supportive young people can challenge the future based on emotional stability and form a positive outlook on themselves and others.

The limitation of this study is that it is limited to generalize to all adolescents because only the research papers for middle, high and high school students are meta-analyzed. Second, in this study, optimism as a personal protective factor, mental health problem as a personal risk 
factor, and empathy as a family protective factor were found to be factors of high effect size close to a large level, but subjective well-being and optimism, Due to the lack of research on each factor between mental health problems and empathy, a small number of articles were included in the analysis. Third, this study analyzed 44 degree papers and 22 journal papers from domestically published research papers. Research papers that were difficult to obtain the original text were excluded from the analysis. In case of analysis including the result of excluded research, there may be some difference in the effect size.

\section{References}

[1] Edward F. Diener and Robert Biswas-Diener, "Will money increase subjective well-being?" A literature review and guide to needed research, Social Indicators Research, vol.57, no.2, pp.119-169, (2002) DOI: 10.1023/A:1014411319119

[2] Edward F. Diener, "Subjective well-being. Psychology Bulletin,” vol.193, pp.542-575, (1984)

[3] Edward F. Diener, "Subjective well-being: The science of happiness, and a proposal for a national index," American Psychologist, vol.55, pp. 34-43, (2000)

[4] K. J. Kang, "Multiple mediated effects of unconditional self acceptance and interpersonal satisfaction in the relations between parenting attitude and subjective well-being in children," M.S. thesis, Chonnam National University, Gwangju, Republic of Korea, (2015)

[5] Y. S. Youm, K. M. Kim, and Y. C. Sung, 2018 Korean Children and Youth Happiness Index International Comparative Research Report, Yonsei University, Institute for social development studies, Seoul, Republic of Korea, (2018)

[6] Christopher Peterson, “A primer in positive Psychology,” New York: Oxford University Press, (2006)

[7] Sonja Lyubomirsky, Laura King, and Edward F. Diener, "The benefits of frequent positive affect: Does happiness Lead to success?" Psychology Bulletin, vol.131, pp.803-855, (2005) DOI: 10.1037/00332909.131.6.803

[8] H. M. Park, "Mediating effects of emotion regulation strategies on the relationship between mindfulness and psychological well-being," Sungshin women's university, Seoul, Republic of Korea, (2011)

[9] H. J. Paek, B. K. Lee, Salmon, Charles T. Salomon, and Kim Witte, "The Contextual Effects of Gender Norms, Communication, and Social Capital on Family Planning Behaviors in Uganda: A multi-level Approach," Health Education \& Behavior, vol.35, no.4, pp.461-477, (2008)

[10] J. H. Park, "Developing and testing of a classroom-based counseling program to enhance the psychological well-being of adolescents," Ph.D. dissertation, Pusan National University, Pusan, Republic of Korea, (2007)

[11] S. S. So, "A study on integration model of psychological well-being and school adjustment in adolescents," Ph.D. dissertation, Kyonggi University, Suwon, Republic of Korea, (2011) 
Meta-Analysis of the Variables Related to Subjective Well-being of Youth

This page is empty by intention. 